\title{
A Study of Maternal and Perinatal Outcomes in Pregnancy with Heart Diseases: Validation of CARPREG II Risk Prediction Index
}

\author{
Deepika Gurnani ${ }^{1}$, Preeti F Lewis ${ }^{2}$, Sana T Bijapur ${ }^{3}$
}

\begin{abstract}
Aim and objective: The aim of the study was to assess efficacy of CARPREG II risk prediction index in predicting poor maternal outcomes in pregnancies with heart diseases.

Materials and methods: This study was conducted in the department of obstetrics and gynecology at a tertiary care center in Mumbai. This was a retrospective study of 66 pregnant women with structural heart diseases. CARPREG II score was calculated for each patient as per history, clinical examination, specific lesion through echo findings, and delivery of antenatal care. Primary and secondary adverse cardiac outcomes were noted during antepartum and postpartum periods. Obstetric and perinatal outcomes were also recorded.

Results: Mean age of the study population was 27.36 years. A total of $51.5 \%(n=34)$ patients were primigravidae. Majority of the subjects about $59.09 \%(n=39)$ had rheumatic heart diseases (RHDs) and $25.75 \%(n=17)$ had congenital heart diseases (CHDs). Overall, vaginal delivery was the most common route with an increased rate of Cesarean section (41.07\%) and instrumental vaginal delivery (16.6\%). There was no significant difference in observed and expected values of primary maternal outcome ( $p$ value $=0.9)$. All the maternal adverse clinical events were more common in groups of patients with CARPREG II index of 4 or more, but in score $<3$, the risk index overestimated maternal adverse cardiac events. Perinatal complications did not show any correlation with CARPREG II score.

Conclusion: Pregnancy with cardiac disease is associated with high maternal and fetal morbidity and mortality. CAREPREG II risk prediction index predicts maternal outcomes effectively and can be used routinely in clinical practices to assess the maternal risks.

Clinical significance: Integration of general cardiac factors and disease-specific factors along with delivery of care predictor emphasizes the role of skilled antenatal care in improving outcomes in pregnancy with cardiac diseases.

Keywords: CARPREG II risk prediction index, Heart disease, Maternal outcome, Perinatal outcome, Pregnancy.

Journal of South Asian Federation of Obstetrics and Gynaecology (2021): 10.5005/jp-journals-10006-1941
\end{abstract}

\section{INTRODUCTION}

Heart diseases affect nearly $1 \%$ of all pregnancies. Indian researchers have recorded incidence of $3.1 \%$ of heart diseases in pregnancy in tertiary care centers. ${ }^{1}$ It causes significant morbidity and mortality. Rheumatic heart disease (RHD) is responsible in majority of cases but incidence of CHD is on the rise from last decade. ${ }^{2,3}$ As with all medical disorders, pre-pregnancy counseling has a major preventive role in women with heart diseases in ensuring an optimal maternal outcome. This calls for the need for a risk index that predicts poor maternal outcomes effectively.

Most studies so far have predicted maternal outcomes and the decision of management based on the patient's New York Heart Association (NYHA) score and/or disease's modified WHO classification (m-WHO class).

The multicenter Cardiac Disease in Pregnancy Study ${ }^{4}$ (CARPREG study) was the first to develop a risk index to predict the likelihood of maternal cardiac complications from general maternal clinical and echocardiographic data obtained during the baseline antepartum visit.

In 2018, CARPREG II study ${ }^{5}$ was published in the Journal of the American College of Cardiology (JACC). A new comprehensive CARPREG II risk prediction index was designed to predict maternal cardiac complications during pregnancy which takes into account

\begin{abstract}
${ }^{1-3}$ Department of Obstetrics and Gynecology, Grant Medical College and JJ Group of Hospitals, Mumbai, Maharashtra, India

Corresponding Author: Deepika Gurnani, Department of Obstetrics and Gynecology, Grant Medical College and JJ Group of Hospitals, Mumbai, Maharashtra, India, Phone: +91 07597450505, e-mail: drdg19nov@gmail.com

How to cite this article: Gurnani D, Lewis PF, Bijapur ST. A Study of Maternal and Perinatal Outcomes in Pregnancy with Heart Diseases: Validation of CARPREG II Risk Prediction Index. J South Asian Feder Obst Gynae 2021;13(4):254-258.
\end{abstract}

Source of support: Nil

Conflict of interest: None

the late first antenatal visit in addition to general and lesion-specific estimators as 10 predictors of adverse events in pregnant women with heart diseases. There are not many studies done in India which incorporated this index to assess pregnant women with heart diseases. We have used this index in our retrospective study on pregnant women with heart diseases.

In women with heart diseases, the risk of fetal and neonatal complications is higher and they follow the pattern similar to maternal and obstetric outcomes. ${ }^{6}$

(c) The Author(s). 2021 Open Access This article is distributed under the terms of the Creative Commons Attribution 4.0 International License (https://creativecommons. org/licenses/by-nc/4.0/), which permits unrestricted use, distribution, and non-commercial reproduction in any medium, provided you give appropriate credit to the original author(s) and the source, provide a link to the Creative Commons license, and indicate if changes were made. The Creative Commons Public Domain Dedication waiver (http://creativecommons.org/publicdomain/zero/1.0/) applies to the data made available in this article, unless otherwise stated. 


\section{Materials and Methods}

\section{Study Design}

This was a retrospective single-center observational study including 66 pregnant women with structural heart diseases.

\section{Study Site}

This study was conducted in the department of obstetrics and gynecology at a tertiary care center in Mumbai, Maharashtra (MH).

\section{Study Timeline}

The duration of the study period was from March 2018 to April 2020.

\section{Aim and Objectives}

The aim of our study was to evaluate the CARPREG II index for risk prediction in pregnancies with heart diseases.

The objectives of our study were as follows:

- To calculate CARPREG II index in all pregnancies with heart diseases during the study period.

- To study maternal complication during antenatal, intranatal, and postnatal periods and find a correlation with risk index.

- To study mode of delivery, outcome of pregnancy, and fetal/ neonatal outcome in pregnancy with heart diseases.

\section{Hypothesis}

$H_{o}$-There is no significant difference between the predicted adverse maternal outcome and observed adverse maternal outcome in pregnancy with heart disease, i.e., CARPREG II study does predict poor maternal outcomes correctly.

$\mathrm{H}_{1}$-There is a significant difference between the predicted adverse maternal outcome and observed adverse maternal outcome in pregnancy with heart disease, i.e., CARPREG II study does not correctly predict poor maternal outcomes.

\section{Selection Criteria}

\section{Inclusion Criteria}

The following are the inclusion criteria used in the present study:

- All pregnant patients with structural heart diseases, managed at our center during the study period.

- Pregnancies with outcomes in the study period.

- All cases with complete records with necessary data available including echo findings.

\section{Exclusion Criteria}

Some of the exclusion criteria of this study were as follows:

All the pregnancies with outcome pending after completion of the study period.

All cases with incomplete records.

\section{Sample Size}

$$
\text { Sample size, } \quad n=N \times \frac{\frac{Z^{2} \times p \times(1-p)}{e^{2}}}{\left[N-1+\frac{Z^{2} \times p \times(1-p)}{e^{2}}\right]}
$$

where

- $N=$ Population size,

- $Z=$ Critical value of the normal distribution at the required confidence level,
- $p=$ Sample proportion,

- $e=$ Margin of error

By the above-mentioned calculation, we obtained the sample size $n=66$.

\section{Data Collection}

We collected data from hospital records in a predesigned proforma. Demographic details, antenatal history, medical history, drug history, past history, examination findings, baseline NYHA class, echo findings, pregnancy outcome in terms of mode of delivery, need and cause of termination, and fetal and maternal complications were noted. Ten predictors as per CARPREG II study ${ }^{5}$ were broadly grouped into three categories:

- Five general cardiac factors:

- prior cardiac events (history of HF, stroke, or transient ischemic attack) or arrhythmia (three points),

- baseline NYHA functional class III or IV or cyanosis (three points),

- high-risk valve lesions/left ventricular outflow tract obstruction (two points),

- systemic ventricular dysfunction (two points),

- absence of prior cardiac interventions (one point);

- Four lesion-specific variables:

- mechanical prosthesis (three points),

- coronary artery disease (two points),

- high-risk aortopathy (two points),

- pulmonary hypertension (two points);

- One variable related to the delivery of care: late pregnancy assessment (one point).

\section{Data Analysis}

Primary maternal outcomes were defined as any of the following: maternal cardiac death, cardiac arrest, sustained arrhythmia requiring treatment, heart failure, stroke or transient ischemic attack, cardiac thromboembolism, myocardial infarction, and vascular dissection.

Secondary outcomes were a decline in NYHA functional class by $\geq 2$ classes during the antepartum period or the need for urgent invasive treatment during pregnancy and up to the sixth postpartum week. These were incorporated from the aforementioned CARPREG II study. ${ }^{5}$

Neonatal complications noted were intrauterine fetal death (IUFD), neonatal death, prematurity, small-for-gestation age birth, low birth weight $(<2.5 \mathrm{~kg})$, intrauterine growth retardation (IUGR), respiratory distress syndrome, intracranial hemorrhage, and CHDs of newborns.

\section{Statistical Analysis}

The data were initially captured into the predesigned proforma and then transferred to Microsoft Excel for analysis. Appropriate statistical tests were applied wherever required.

\section{RESULTS}

In the present study, we analyzed the data of 66 pregnant women with heart diseases who were managed at our center. Majority of the subjects about $59.09 \%(n=39)$ had RHDs and $25.75 \%$ $(n=17)$ had CHDs. There were $9.09 \%(n=6)$ cases of peripartum 
cardiomyopathy and $6.06 \%(n=4)$ cases of LV dysfunction secondary to hypothyroidism, hypertension, and myocarditis.

The demographic details are shown in Table 1. Mean age of the study population was 27.36 years. Age range varied from 19 to 39 years. Patients were grouped in five groups as per CARPREG II score, $0-1,2,3,4$, and more than 4 . A maximum number of patients (36.4\%) had CARPREG score of $0-1$, while CARPREG II scores of more than 4 in $30 \%$ of the patients.

Table 2 describes obstetric outcomes in the present study. Vaginal delivery was the most common route of delivery in $50 \%$ of the patients $(n=33)$. Cesarean delivery rate (number of cesarean section (CS) performed per 100 deliveries in women with heart disease) was $41.07 \%$. About $15.15 \%(n=10)$ of the pregnancies were aborted, of which $60 \%$ were terminated due to cardiac indication, 20\% due to other causes like contraceptive failure and use of teratogenic drugs in the first trimester, and two cases had spontaneous abortion.

Figure 1 shows maternal adverse outcome according to CARPREG II scores. Totally, 17 primary adverse cardiac events inclusive of $4.5 \%(n=3)$ maternal deaths, $16.6 \%(n=11)$ heart failure, and $4.5 \%(n=3)$ stroke/TIA were observed. Among 26 secondary cardiac events, $28.8 \%(n=19)$ of the cases had worsening in NYHA score by $\geq 2$ classes and $10.6 \%(n=7)$ of the cases needed invasive treatment in antepartum or postpartum period up to the sixth week. All the maternal adverse clinical events were more in patients who had CARPREG II index 4 or more. Among patients with CAREPREG II INDEX of more than 4, 30\% had heart failure, $15 \%$ had stroke, $10 \%$ died, $50 \%$ had worsening of NYHA, and $20 \%$ needed surgery during antenatal or postnatal period within 6 weeks. However, there was no case of cardiac thromboembolism, myocardial infarction, and vascular dissection.

Table 1: Demographic details of the study $(n=66)$

\begin{tabular}{lrc}
\hline Attribute & No. of patients & Percentage (\%) \\
\hline Age & 3 & \\
$<20$ & 60 & 4.5 \\
$20-35$ & 3 & 90.9 \\
$>35$ & & 4.5 \\
Gravida score & 34 & \\
Primigravida & 32 & 51.5 \\
Multigravida & & 48.5 \\
Referral status & 32 & \\
Referred & 34 & 48.5 \\
Nonreferred & & 51.5 \\
Antenatal clinic (ANC) visit & 18 & \\
Delayed first visit after 20 weeks & & 27.3 \\
NYHA class at first visit & 44 & \\
I & 16 & 66.6 \\
II & 6 & 24.2 \\
III & Nil & 9.09 \\
IV & & 0 \\
CARPREG II score & 24 & \\
0-1 & 7 & 36.4 \\
2 & 8 & 10.6 \\
3 & 6 & 12.4 \\
4 & 21 & 30.6 \\
More than 4 & &
\end{tabular}

As shown in Table 3, we found a higher incidence of poor maternal outcome in CARPREG index 4 or more, as predicted. But we observed a lower incidence of adverse events in CARPREG II group 0-1 and 3 than predicted by CARPREG II index. Chi-square $=0.99, p$ value $=0.9$ which means the null hypothesis is true, i.e., there is no significant difference between the predicted adverse maternal outcome and observed maternal outcome in pregnancies with heart diseases. So we can say, CARPREG II study does predict poor maternal outcomes correctly.

There were three cases of maternal deaths. One, 29-yearold G2P1, a referred case of ventricular septal defect (VSD) with Eisenmenger syndrome, had CARPREG II index of 7 and died in the immediate postpartum period. The cause of death was (VSD) with Eisenmenger syndrome with polycythemia with thrombocytopenia. The other contributory factor was inadequate antenatal care, which was avoidable with proper counseling regarding grave outcome of this cardiac condition and need of termination.

Other two cases of maternal mortality were due to postpartum cardiomyopathy. A P1L1, 21 years old presented with features of heart failure on day 6 postpartum, 2D-echo, showed ejection fraction (EF) of $25 \%$. She was managed for heart failure in ICU but succumbed on day 19. Another case P3L3, 31 years old presented with heart failure on day 2 postpartum with EF of $25 \%$, was succumbed on day 12 postpartum.

In the present study, perinatal complications were studied $(n=56)$. Abortions were excluded from calculations. The most common complications were low birth weight (31.7\%) followed by preterm delivery (25.3\%), oligohydramnios (9.5\%), and IUGR (7.9\%). There were four perinatal deaths including two IUFD and two neonatal deaths attributed to low birth weight, IUGR, and its complications. Perinatal complications did not show any correlation with CARPREG II score. However, all complications were more frequent in WHO class IV.

Table 2: Obstetric outcome in the present study

\begin{tabular}{lcc}
\hline Outcome & No. of patients & Percentage (\%) \\
\hline Mode of delivery ( $n=66)$ & 22 & 33.3 \\
Normal vaginal delivery & 11 & 16.6 \\
Assisted vaginal delivery & 23 & 34.8 \\
Cesarean section (CS) & 10 & 15.2 \\
Abortions & & \\
Indication of CS & 18 & 78.3 \\
CS for obstetric indication & 5 & 21.7 \\
CS for cardiac indication & & \\
Urgency of CS & 7 & 30.4 \\
Elective CS & 16 & 69.5 \\
Emergency CS & & \\
Instrumental vaginal delivery & 10 & 90.9 \\
Vacuum assisted VD & 1 & 9.09 \\
Forceps assisted VD & & \\
Gestational age at delivery $(n=56)$ & 17 & 30.4 \\
$<37$ weeks & 39 & 69.6 \\
$\geq 37$ weeks & & 60 \\
Abortions ( $n=10)$ & 6 & 20 \\
MTP/cardiac disease & 2 & \\
MTP/other indications & 2 & \\
Spontaneous abortion & & \\
\hline & & \\
\hline
\end{tabular}




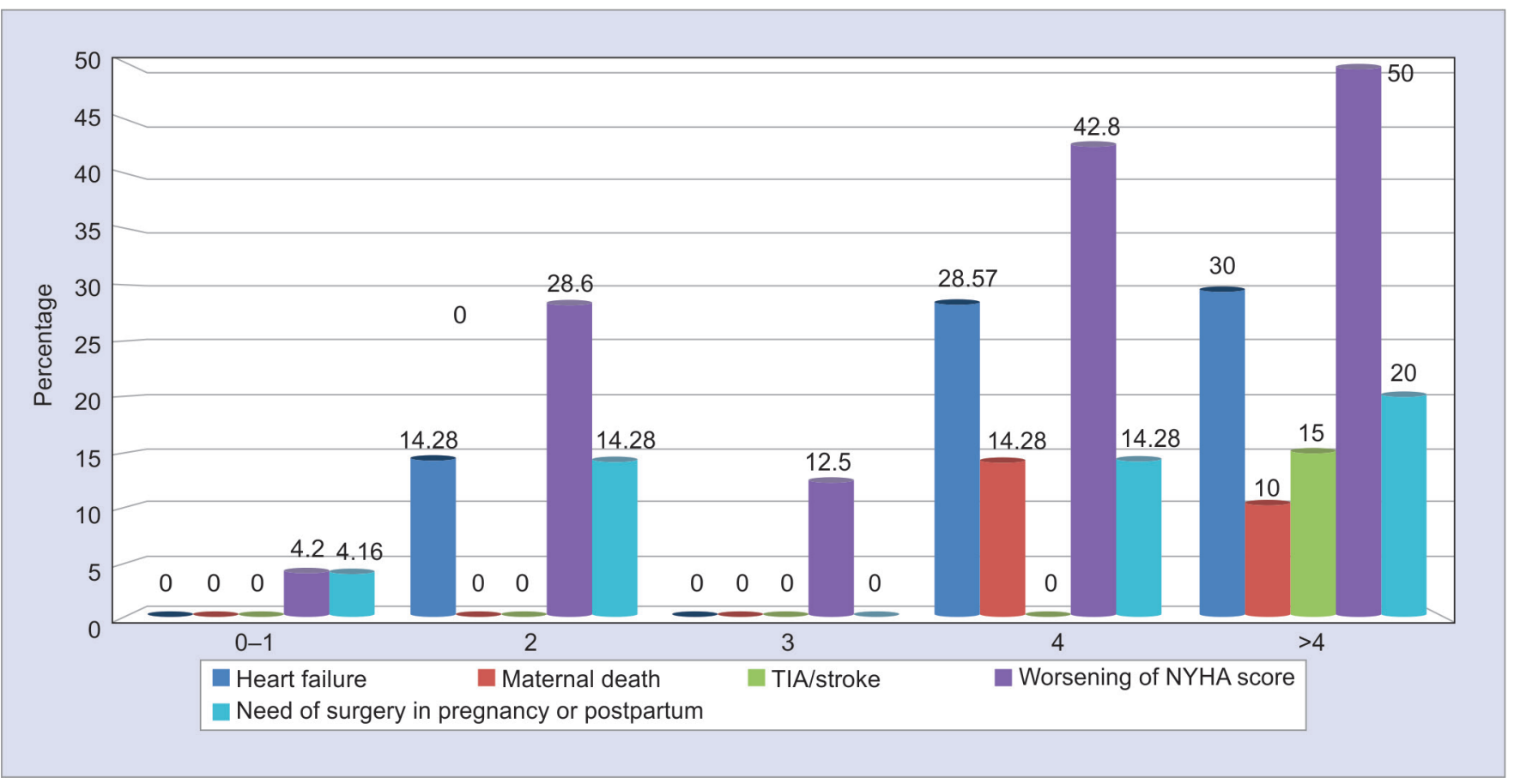

Fig. 1: CARPREG II index vs maternal outcome

Table 3: Risk of primary cardiac outcome (predicted vs observed)

\begin{tabular}{lcc}
\hline CARPREG II index & Predicted (CARPREG II study) (\%) & Observed (present study) (\%) \\
\hline $0-1$ & 5 & 0 \\
2 & 10 & 14.2 \\
3 & 15 & 0 \\
4 & 22 & 28 \\
$>4$ & 41 & 45 \\
\hline$p=0.9$ & &
\end{tabular}

\section{Discussion}

Our study is one of the first few in India, to study the validation of CARPREG II index for assessment of pregnancy outcomes in women with heart diseases. Women with heart diseases suffer significantly high mortality and morbidity than general population. There are a few scores to interpret the risk, like $\mathrm{m}-\mathrm{WHO}$ classification of cardiac disease, CARPREG I index, ZAHARA I risk index ${ }^{6}$ for CHDs, but none of them took into account delayed antenatal care as a risk parameter which was considered in CARPREG II study.

The RHDs are still the most common heart diseases in developing world including India. But, the incidence of CHDs is rising in obstetric population, which is as a result of improved health care and increased survival of CHD patients into the reproductive age group. ${ }^{7}$ We found $62 \%$ incidence of RHDs and $27 \%$ CHDs which are consistent with Subbaiah et al. ${ }^{8}$ (63\% RHDs $36 \%$ CHDs). While Agrawal et al. ${ }^{2}$ from King George's Medical University (KGMC), Lucknow, found a higher incidence of RHDs (93.5\%) which may be because of variations in geographic distribution and ethnicity of the study population.

Mean age of our study population was 27.36 years. Agrawal et al. ${ }^{2}$ recorded a majority (83\%) of the subjects in age group less than 30 years. Salam et al. ${ }^{9}$ found a maximum number of patients in $20-30$ years of age group ( $74.5 \%)$.
Lumsden et al. ${ }^{10}$ studied validation of CARPREG I index and found that the rates of adverse cardiac events and neonatal events observed in the study were higher than those predicted by existing CARPREG I and $\mathrm{m}-\mathrm{WHO}$ risk models. Martins et al. ${ }^{11}$ studied the validation of CARPREG I index in the Brazilian population and observed that CARPREG index overestimated the risk in the group with score of 1 or more and underestimated the risk in CARPREG 0 score group. We found that all the maternal adverse clinical events were more common in groups of patients with CARPREG II index of 4 or more, but in score $<3$, the risk index overestimated maternal adverse cardiac events.

Obstetric and adverse maternal outcomes are compared in Table 4. The CS rates were found to be higher than the general population in almost all the studies, and the majority were due to obstetric indication. Instrumental delivery rates were also higher.

We recorded $12.6 \%$ incidence of heart failure which was fairly higher than CARPREG II study, likely because of a difference in healthcare structure and population ethnicity, and it was comparable to Salam et al., ${ }^{9}$ Joshi et al., ${ }^{12}$ Lumsden et al., ${ }^{10}$ and Agrawal et al., ${ }^{2}$ while lower than Beaton et al. ${ }^{13}$ Konar and Chaudhuri $^{14}$ in a large multicentric study in India showed a lower incidence of all maternal complications.

We reported $25.3 \%$ preterm pregnancy rate lower than other studies (Joshi et al. ${ }^{12} 42.1 \%$, Agrawal et al. ${ }^{2} 62.7 \%$, Lumsden 
Table 4: Comparisons of obstetric and adverse maternal outcomes

\begin{tabular}{|c|c|c|c|c|c|c|c|c|}
\hline Outcome & $\begin{array}{c}\text { Present } \\
\text { study (\%) }\end{array}$ & $\begin{array}{l}\text { CARPREG II } \\
\text { study (\%) }\end{array}$ & $\begin{array}{c}\text { Konar } \\
\text { et al. (\%) }\end{array}$ & $\begin{array}{c}\text { Salam } \\
\text { et al. }{ }^{9}(\%)\end{array}$ & $\begin{array}{l}\text { Lumsden } \\
\text { et al. (\%) }\end{array}$ & $\begin{array}{c}\text { Joshi } \\
\text { et al. (\%) }\end{array}$ & $\begin{array}{l}\text { Agrawal } \\
\text { et al. (\%) }\end{array}$ & $\begin{array}{l}\text { Beaton } \\
\text { et al. (\%) }\end{array}$ \\
\hline Cesarean section rate & 41.07 & & 33 & 36.7 & 15.4 & 23.8 & 38 & \\
\hline Instrumental VD rate & 16.6 & & 17 & & & 4.8 & 39.2 & \\
\hline MTP rate & 12.1 & & & 10 & & 19 & & \\
\hline Heart failure & 12.6 & 6.2 & 7.4 & 11.1 & 9.3 & 16.7 & 9.2 & 33.9 \\
\hline Stroke/cerebral infarct & 4.7 & 0.7 & 0.3 & & 0 & & 0.92 & \\
\hline Maternal death & 4.5 & 0.3 & 1.1 & 4.44 & 9.3 & 4.8 & 3.7 & 15.4 \\
\hline NYHA worsening & 28.8 & & 6.4 & 11.1 & & 33 & & \\
\hline Need of surgery in pregnancy/postpartum period & 10.6 & & 4.27 & & & & & \\
\hline
\end{tabular}

et al. ${ }^{10} 41.6 \%$ ) and $31.7 \%$ low birth weight (Agrawal et al. ${ }^{2} 16 \%$, Lumsden et al. ${ }^{10}$ 30.4\%), and 7.9\% IUGR (Manh et al. ${ }^{15}$ 9.15\%). Our perinatal mortality rate was $4.7 \%$. Konar and Chaudhuri ${ }^{14}$ reported perinatal mortality rate of $4 \%$, Agrawal et al. ${ }^{2}$ reported stillbirth rate of $7.8 \%$, and Salam et al. ${ }^{9}$ reported neonatal death (NND) rate of $2.6 \%$.

\section{Limitations}

Limitation of the present study is largely related to its retrospective design. The study lacks matching control population.

\section{Conclusion and Recommendation}

Overall, women with heart diseases have poor maternal and perinatal outcomes than general population, but it can be improved with early assessment in prepregnancy and antenatal period, and pregnancy should be allowed only after due correction of the heart defect and clinical optimization. In many patients, structural heart disease is diagnosed in antenatal period only, when women come for routine visit. After diagnosis of heart diseases, patients should be assessed by CARPREG II risk index and explained about the outcome. A multidisciplinary approach is needed in the cases of pregnancy with heart disease to determine the mode of delivery, timing of delivery, change in anticoagulant drugs in pregnancy, type of anesthesia, care of neonate, and follow-up of mother for better maternal and fetal outcomes.

In the future, more studies are needed for validation of CARPREG II index in the Indian population.

\section{References}

1. Bhatla N, Lal S, Behera G, et al. Cardiac disease in pregnancy. Int J Gynaecol Obstet 2003;82(2):153-159. DOI: 10.1016/s00207292(03)00159-0.

2. Agrawal S, Agrawal A, Bhandari M, et al. Critical analysis of all pregnancies with heart disease, misses and near misses over 1-year period along with expert group so as to optimize outcome and improve patient care - need-based analysis. Heart India 2019;7(2):5562. DOI: 10.4103/heartindia.heartindia_15_19.
3. Selvarani G, Sivakumar GS, Swaminathan N, et al. Prevalence study on heart diseases among antenatal mothers. Int J Sci Stud 2017;5(5): 204-208. DOI: 10.17354/ijss/2017/423.

4. Siu SC, Sermer M, Colman JM, et al. Prospective multicenter study of pregnancy outcomes in women with heart disease. Circulation 2001;104(5):515-521. DOI: 10.1161/hc3001.093437.

5. Silversides CK, Grewal J, Mason J, et al. Pregnancy outcomes in women with heart disease: the CARPREG II study. J Am Coll Cardiol 2018;71(21):2419-2430. DOI: 10.1016/j.jacc.2018.02.076.

6. Drenthen W, Boersma E, Balci A, et al. Predictors of pregnancy complications in women with congenital heart disease. Eur Heart J 2010;31(17):2124-2132. DOI: 10.1093/eurheartj/ehq200.

7. Owens A, Yang J, Nie L, et al. Neonatal and maternal outcomes in pregnant women with cardiac disease. J Am Heart Assoc 2018;7(21):e009395. DOI: 10.1161/JAHA.118.009395.

8. Subbaiah M, Sharma V, Kumar S, et al. Heart disease in pregnancy: cardiac and obstetric outcomes. Arch Gynecol Obstet 2013;288(1): 23-27. DOI: 10.1007/s00404-013-2730-2.

9. Salam S, Mushtaq S, Mohi-ud-Din K, et al. Maternal and fetal outcome in pregnancy with heart disease in tertiary care hospital in India. Int J Reprod Contracept Obstet Gynecol 2017;6(9):3947-3951. DOI: 10.18203/2320-1770.ijrcog20174041.

10. Lumsden R, Barasa F, Park LP, et al. High burden of cardiac disease in pregnancy at a national referral hospital in Western Kenya. Glob Heart 2020;15(1):10. DOI: 10.5334/gh.404.

11. Martins LC, Freire CM, Capuruçu CA, et al. Risk prediction of cardiovascular complications in pregnant women with heart disease. Arq Bras Cardiol 2016;106(4):289-296. DOI: 10.5935/abc.20160028.

12. Joshi G, Joshi SC, Jha SK, et al. Maternal heart disease and pregnancy outcome: findings from a retrospective cohort in a tertiary care government hospital in Haldwani, Nainital. Nig J Cardiol 2015;12(2):120-123. DOI: 10.4103/0189-7969.152027.

13. Beaton A, Okello $E$, Scheel $A$, et al. Impact of heart disease on maternal, fetal and neonatal outcomes in a low-resource setting. Heart 2019;105(10):755-760. DOI: 10.1136/heartjnl-2018-313810.

14. Konar H, Chaudhuri S. Pregnancy complicated by maternal heart disease: a review of 281 women. J Obstet Gynaecol India 2012;62(3):301-306. DOI: 10.1007/s13224-012-0220-2.

15. Nguyen Manh T, Bui Van N, Le Thi H, et al. Pregnancy with heart disease: maternal outcomes and risk factors for fetal growth restriction. Int J Environ Res Public Health 2019;16(12):2075. DOI: 10.3390/ijerph16122075. 